\title{
Vasohibin2 promotes adriamycin resistance of breast cancer cells through regulating ABCG2 via AKT signaling pathway
}

\author{
DELIANG MA ${ }^{1}$, LIANG WU ${ }^{1}$, SHUANGJIE $\mathrm{LI}^{2}$, ZHIGANG SUN ${ }^{3}$ and KAI WANG ${ }^{2}$ \\ ${ }^{1}$ Department of Oncology, Linyi Central Hospital, Linyi, Shandong 276400; ${ }^{2}$ Department of Breast Surgery, \\ Weifang People's Hospital, Weifang, Shandong 261041; ${ }^{3}$ Central Laboratory, \\ Linyi Central Hospital, Linyi, Shandong 276400, P.R. China
}

Received March 21, 2017; Accepted September 27, 2017

DOI: $10.3892 / \mathrm{mmr} .2017 .7792$

\begin{abstract}
As a well-known angiogenic factor in different histology and pathological conditions, the pro-progressive role of vasohibin2 (VASH2) has been reported in various types of tumors. However, its role in drug resistance of breast cancer has not been reported so far. The present study demonstrated that MCF-7 cells with increased expression of VASH2 demonstrate stronger adriamycin (ADM) resistance compared with MDA-MB-231 cells with decreased expression of VASH2. Overexpression of VASH2 in MDA-MB-231 cells increased ADM resistance and silencing VASH2 in MCF-7 cells inhibited ADM resistance. Furthermore, in newly established ADM resistant cell lines, VASH2 was significantly upregulated. These results revealed the promotive role of VASH 2 in the ADM resistance of breast cancer cells. In addition, overexpression of VASH2 in MDA-MB-231 cells significantly upregulated ATP-binding cassette sub-family $\mathrm{G}$ member 2 (ABCG2), however silencing VASH2 in MCF-7 cells inhibited ABCG2 significantly. Silencing ABCG2 abrogated increase of ADM resistance induced by VASH2 overexpression in MDA-MB-231 cells. This proved that VASH2 induced ADM resistance through promoting expression of ABCG2, at least in part. Further study regarding the underlying molecular mechanism demonstrated that VASH2 promoted ABCG2 via the protein kinase $\mathrm{B}$ (AKT) signaling pathway. Overall, VASH2 may promote drug resistance of breast cancer cells through regulating ABCG2 via the AKT signaling pathway. This suggests a novel therapeutic target to inhibit drug resistance in breast cancer, for a more efficient therapeutic outcome.
\end{abstract}

Correspondence to: Mr Kai Wang, Department of Breast Surgery, Weifang People's Hospital, 151 Guangwen Street of Kuiwen, Weifang, Shandong 261041, P.R. China

E-mail:wangkaiatweifang@163.com

Key words: vasohibin2, breast cancer, drug resistance, adriamycin, ATP-binding cassette sub-family G member 2

\section{Introduction}

Breast cancer (BC) is the most common female malignant tumors in the world and its incidence is rising annually (1). In recent years, as development of treatment modalities and early detections, overall survival of $\mathrm{BC}$ patients has been improved to some extent. However, for the occurrence of drug resistance, the prognosis of some patients is still not good (2-5). So, to find effective targets for inhibiting drug resistance is urgent and will contribute to better therapy outcome of BC patients.

Vasohibin2 (VASH2) is a member of vasohibin family and initially known as an angiogenic factor in different histology and pathology conditions (6). In recent years, its roles in different tumors have been widely studied. It is reported that VASH2 can play important roles in proliferation, angiogenesis and epithelial mesenchymal transition (EMT) of hepatocellular carcinoma, ovarian adenocarcinoma, pancreatic ductal adenocarcinoma and endometrial cancer cells (7-11). In BC, it has been reported that VASH2 could promote EMT and proliferation of $\mathrm{BC}$ cells through in vivo and in vitro experiments $(12,13)$. However, its role in drug resistance of BC is still unknown.

In the present study, we detected VASH2 expression and drug resistance of different $\mathrm{BC}$ cell lines. We proved that VASH 2 could promote drug resistance of $\mathrm{BC}$ cells through regulating expression of ATP-binding cassette sub-family $\mathrm{G}$ member 2 (ABCG2), at least partly. Moreover, we confirmed that VASH2 could promote expression of ABCG2 via AKT signal pathway.

\section{Materials and methods}

Cell lines and cell culture. Human BC cell lines MCF-7 and MDA-MB-231 were both purchased from American Type Culture Collection (Manassas, VA, USA). Both cell lines were cultured in DMEM medium supplemented with $10 \%$ fetal bovine serum (FBS), at $37^{\circ} \mathrm{C}$, in $5 \% \mathrm{CO}_{2}$.

Stable transfection. Plasmids PcDNA3.1/VASH2-vector, p-GPU6/VASH2-shRNA and control plasmids (Shanghai GenePharma Co., Ltd, Shanghai, China) were transfected into BC cells using Lipo2000 (Invitrogen, Carlsbad, CA, USA) to upregulate or silence VASH2 expression. After $48 \mathrm{~h}$, cells 
Table I. Primary antibodies used in western blot analysis.

\begin{tabular}{ll}
\hline Name & Company \\
\hline VASH2 & Abcam, Cambridge, MA, USA \\
ABCG2 & Abcam, Cambridge, MA, USA \\
Phospho-ERK1/2 & Cell Signaling Technology, Danvers, MA, USA \\
ERK1/2 & Cell Signaling Technology, Danvers, MA, USA \\
Phospho-AKT & Cell Signaling Technology, Danvers, MA, USA \\
AKT & Cell Signaling Technology, Danvers, MA, USA \\
GAPDH & Epitomics, Burlingame, CA, USA
\end{tabular}

VASH2, vasohibin2; ABCG2, TP-binding cassette sub-family G member 2; AKT, protein kinase B.

were cultured in medium containing $1.0 \mathrm{ug} / \mathrm{ml}$ puromycin for 3 weeks, then, monoclone was selected. VASH2 expression was detected by RT-PCR and western blot analysis.

Transitent transfection. To silence ABCG2 expression, siRNA (Shanghai GenePharma Co.) for ABCG2 was transfected into cells using Lipo2000 (Invitrogen). 48 h later, ABCG2 expression was detected by RT-PCR and western blot.

Reverse transcriptase-polymerase chain reaction (RT-PCR). Total RNA was extracted by TRIzol (Invitrogen) according to the manufacturer's instruction. cDNAs were synthesized using PrimeScript RT-PCR kit (TaKaRa, Dalian, China) as protocol. The primers used were as follows: VASH2 forward, 5'-CTC TTCCAGCCTTCCTTCCT-3' and reverse, 5'-AGCACTGTG TTGGCGTACAG-3'; ABCG2 forward, 5'-CTGAGATCC TGAGCCTTTGG-3' and reverse, 5'-TGCCCATCACAACAT CATCT-3'. GAPDH was used as an internal control.

Western blotanalysis. Cell protein was extracted using RIPA lysis buffer. Equal amount of protein was separated by SDS-PAGE and transferred to polyvinylidene difluoride (PVDF) membrane. The membrane was incubated in primary antibodies (Table I) at $4^{\circ} \mathrm{C}$ overnight and in horseradish peroxidase-conjugated secondary antibody (1:5,000; Proteintech Group, Inc, Wuhan, China) in room temperature for $1 \mathrm{~h}$, signal on the membrane was visualized using enhanced chemiluminescence reagents (Pierce, Rockford, IL, USA).

Establishment of cells with stable adriamycin (ADM) resistance. MDA-MB-231 or MCF-7 cells were cultured in DMEM medium containing 0.4 or $0.8 \mathrm{umol} / \mathrm{l} \mathrm{ADM}$ for $48 \mathrm{~h}$, then cells were cultured in ADM free medium. The cells would not be passaged until they grow up to $80 \%$ confluence. ADM concentration is elevated gradually until cells could grow steadily in 1 or 3 umol/l. New drug-resistance cells were named as MDA-MB-231-ADM or MCF-7-ADM.

Drug resistance assay. Cells were plated in 96-well plate in triplicate in DMEM supplemented with 10\% FBS at 8,000 cells per well. After $24 \mathrm{~h}$, the medium was replaced using DMEM containing different concentrations of ADM $(0.02$, $0.08,0.32,1.28,5.12$ and $20.48 \mu \mathrm{mol} / \mathrm{l})$. After $48 \mathrm{~h}, \mathrm{MTT}$ assay was performed at $490 \mathrm{~nm}$ wavelengths. The survival curves were constructed and 50\% inhibitory concentration (IC50) was calculated. The experiment was repeated at least three times.

Statistical analysis. The data were stored and analyzed using SPSS 13.0 software (SPSS Inc., Chicago, IL, USA). The difference between two groups was analyzed using Student two-tailed $\mathrm{t}$ test. IC50 was gotten using regression analysis. $\mathrm{P}<0.05$ was considered to indicate a statistically significant difference.

\section{Results}

MCF-7 cells with higher VASH2 than MDA-MB-231 cells and showed stronger ADM resistance. As shown in Fig. 1A, expression of VASH2 was detected using RT-PCR and western blot, the results showed that VASH2 in MCF-7 cells was much higher than in MDA-MB-231 cells at RNA (A) and protein (B) levels, with significant difference. Drug resistance analysis showed that MCF-7 cells $($ IC 50 $=1.14 \pm 0.12 \mathrm{umol} / \mathrm{l}$ ) exhibited higher survival rate in ADM than MDA-MB-231 cells (IC50 $=0.55 \pm 0.07 \mathrm{umol} / \mathrm{l})(\mathrm{C})$. The results reminded us that VASH2 may affect ADM resistance of $\mathrm{BC}$ cells.

VASH2 promoted ADM resistance of BC cells. To confirm whether VASH2 could affect ADM resistance of BC cells, we changed expression of VASH2 through stable transfection (Fig. 2). After VASH2 was upregulated in MDA-MB-231cells (Fig. 2A), survival rate of cells in ADM increased significantly (Fig. 2C), IC50 increased from $0.41 \pm 0.06$ to $1.18 \pm 0.09 \mathrm{umol} / 1$, with significant difference. Reversely, after VASH2 was silenced in MCF-7 cells (Fig. 2B), survival rate of cells in ADM declined significantly, IC50 declined from 1.31 \pm 0.11 to $0.48 \pm 0.07 \mathrm{umol} / 1$, with significant difference (Fig. 2D). Besides, after cultured in ADM gradually, we got new drug resistance cells MCF-7-ADM and MDA-MB-231-ADM with significantly increased survival rate and IC50 than parent cells (Fig. 2E, F). Western blot showed that VASH2 in MCF-7-ADM cells was significantly higher than in parent cells (Fig. 2G), and it was also the case for MDA-MB-231-ADM cells (Fig. 2H). These results proved the promotion roles of VASH2 in ADM resistance of BC cells.

ABCG2 took part in drug resistance induced by VASH2 in $B C$ cells. As shown in Fig. 3, after VASH2 in MDA-MB-231 

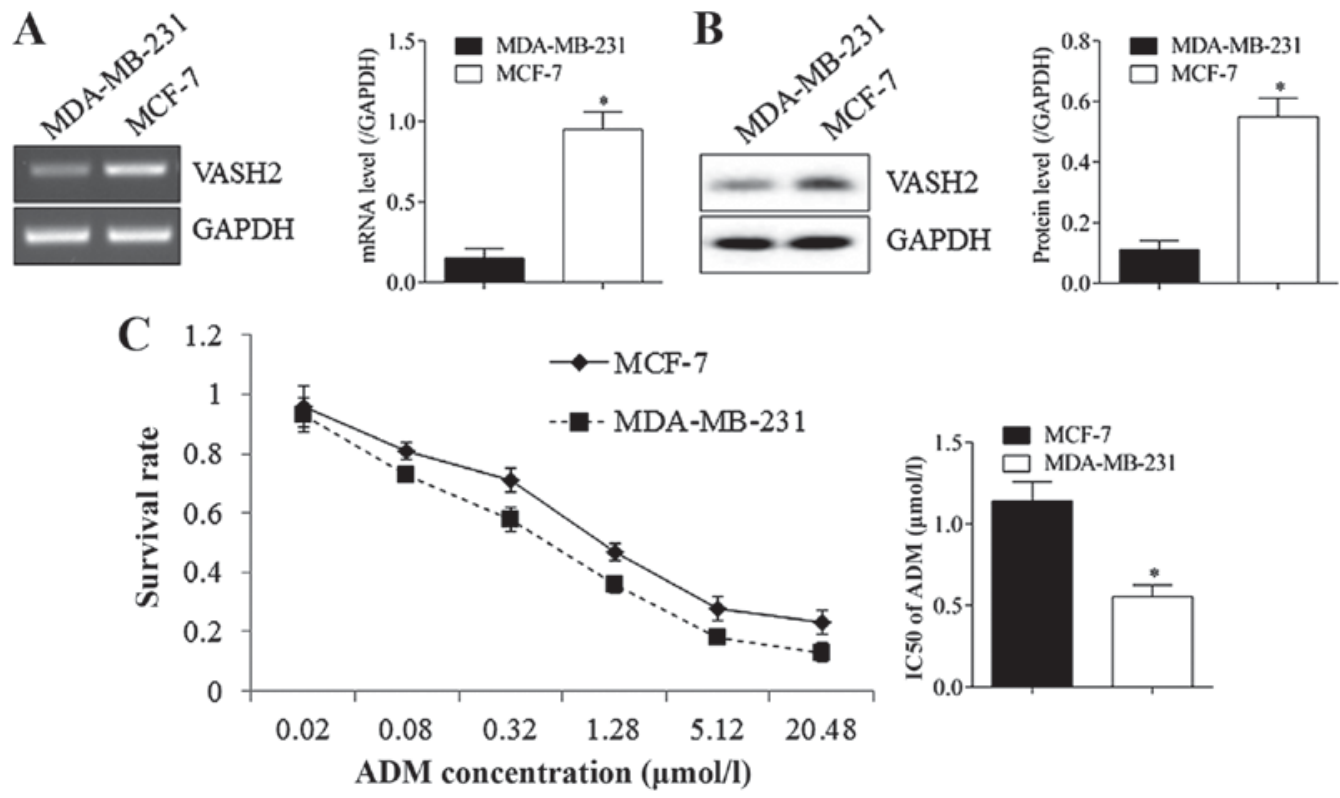

Figure 1. VASH2 expression and ADM resistance of BC cells. VASH2 in MDA-MB-231 was compared to MCF-7 cells both at mRNA (A) and protein (B) levels. (C) Drug resistance ability of MDA-MB-231 was compared to MCF-7 cells. P<0.05 was significant. VASH2, vasohibin2; ADM, adriamycin; BC, breast cancer.

A

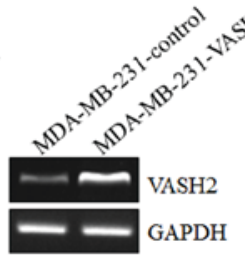

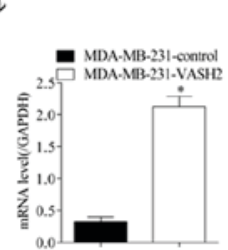

$\mathrm{B}$
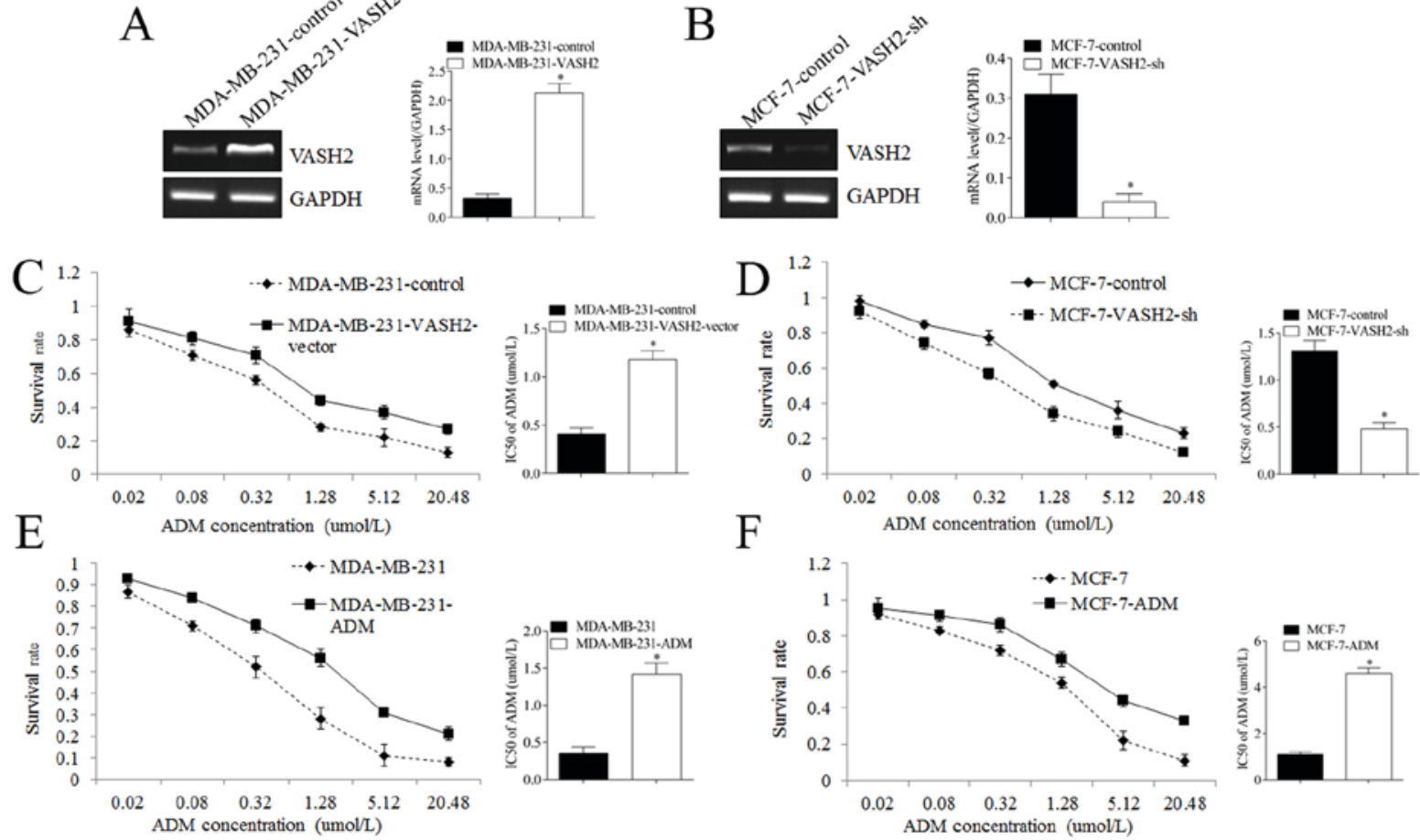

$\mathrm{F}$
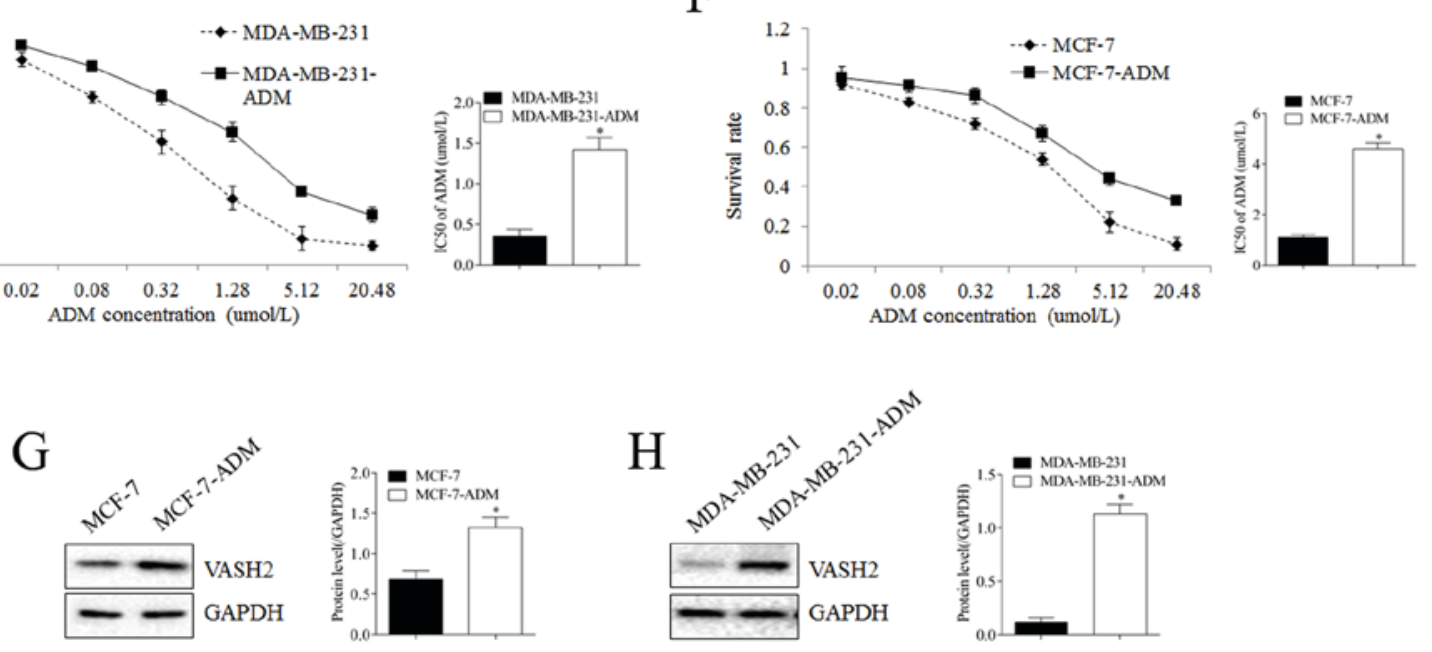

Figure 2 VASH2 promotes drug resistance of BC cells. After VASH2 was overexpressed in MDA-MB-231 cells (A), survival rate and IC50 of ADM increased significantly compared to control cells (C). After VASH2 was silenced in MCF-7 cells (B), survival rate and IC50 declined significantly compared to control cells (D). In newly established ADM resistance cells MDA-MB-231-ADM (E) and MCF-7-ADM (F), survival rate and IC50 increased significantly compared to parent cells. In MCF-7-ADM (G) and MDA-MB-231-ADM cells (H), VASH2 protein was upregulated significantly compared to parent cells. P $<0.05$ was significant. $\mathrm{P}<0.05$ was significant. VASH2, vasohibin2; ADM, adriamycin; BC, breast cancer; IC50, 50\% inhibitory concentration. 

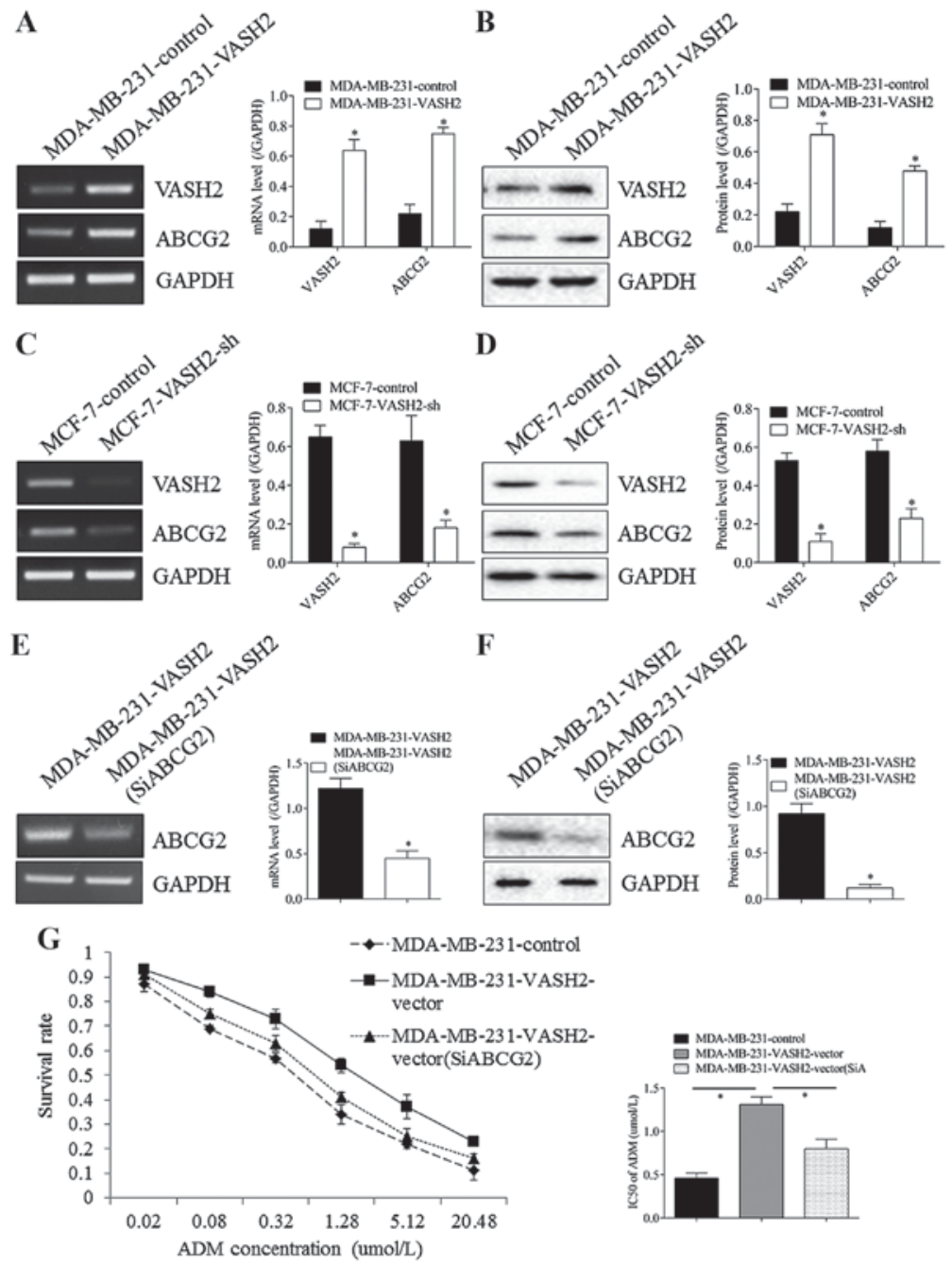

Figure 3. VASH2 promotes drug resistance through regulating expression of ABCG2 After VASH2 was overexpressed in MDA-MB-231 cells, ABCG2 was upregulated at RNA (A) and protein (B) levels compared to control cells. After VASH2 was silenced in MCF-7 cells, ABCG2 was silenced at RNA (C) and protein (D) levels compared to control cells. After ABCG2 in MDA-MB-231-VASH2 cells was silenced by SiRNA at RNA (E) and protein (F) levels, survival rate and IC50 of ADM induced by VASH2 declined significantly compared to control cells (G). P<0.05 was significant. VASH2, vasohibin2; ADM, adriamycin; IC50, 50\% inhibitory concentration.
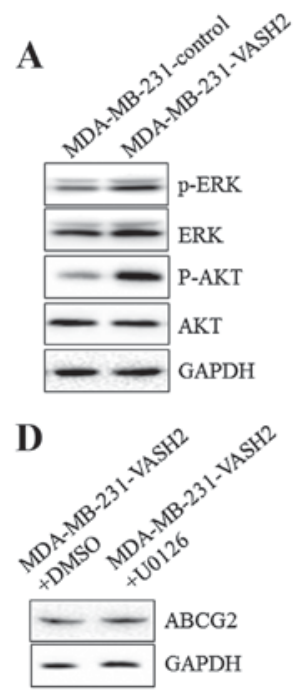

B

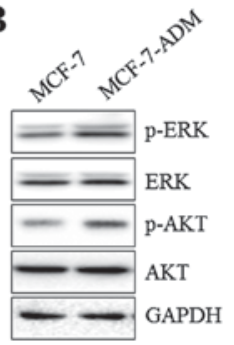

$\mathbf{E}$

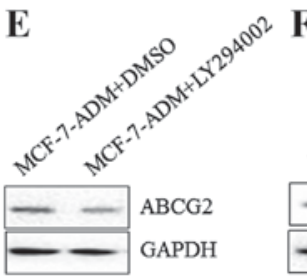

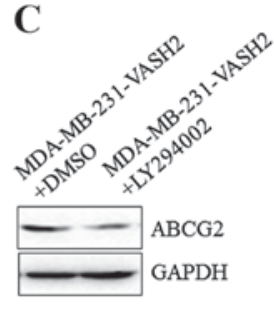

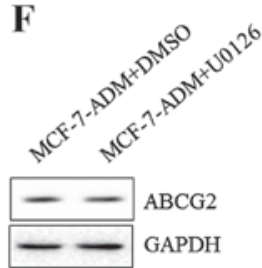

Figure 4. VASH2 promotes expression of ABCG2 via AKT signal pathway. After VASH2 was overexpressed in MDA-MB-231 cells, phosphorylation of AKT and ERK was stimulated significantly compared to control cells (A). In newly established ADM resistance cells MCF-7-ADM, phosphorylation of AKT and ERK was also stimulated significantly compared to parent cells (B). In MDA-MB-231-VASH2 cells, after AKT inhibitor LY294002 (C) or ERK inhibitor U0126 (D) was added, ABCG2 was detected. In MCF-7-ADM cells, after AKT inhibitor LY294002 (E) or ERK inhibitor U0126 (F) was added, ABCG2 was detected. $\mathrm{P}<0.05$ was significant. VASH2, vasohibin2; AKT, protein kinase B. 
cells was overexpressed, expression of ABCG2 was increased both at RNA (Fig. 3A) and protein (Fig. 3B) levels, with significant difference. Reversely, after VASH2 in MCF-7 cells was silenced, expression of ABCG2 was decreased both at RNA (Fig. 3C) and protein (Fig. 3D) levels, with significant difference. Moreover, after ABCG2 in MDA-MB-231-VASH2 cells was silenced by transfection at RNA (Fig. 3E) and protein (Fig. 3F) levels, survival rate declined significantly, but still higher than MDA-MB-231 cells, IC50 declined from $1.31 \pm 0.09$ to $0.81 \pm 0.11 \mathrm{umol} / 1$, with significant difference, but still higher than MDA-MB-231 cells $(0.46 \pm 0.06 \mathrm{umol} / \mathrm{l})$, with significant difference (Fig. $3 \mathrm{G}$ ). These results proved that VASH2 could promote ADM resistance of BC cells through regulating $\mathrm{ABCG} 2$, at least partly.

VASH2 could promote expression of ABCG2 via AKT signal pathway. To confirm potential molecular mechanism, further cell experiments were performed. As shown in Fig. 4A, overexpressing VASH2 stimulated phosphorylation of AKT and ERK in MDA-MB-231 cells significantly. Moreover, phosphorylation of AKT and ERK in MCF-7-ADM cells was also significantly higher than in parent cells (Fig. 4B). Moreover, after AKT inhibitor LY294002 was added, the increase of ABCG2 in MDA-MB-231-VASH2 (Fig. 4C) and MCF-7-ADM (Fig. 4E) cells was inhibited significantly. However, after ERK inhibitor U0126 was added, the increase of ABCG2 in MDA-MB-231-VASH2 (Fig. 4D) or MCF-7-ADM (Fig. 4F) cells did not change. All the results proved that VASH2 could promote expression of ABCG2 in BC cells via AKT signal pathway.

\section{Discussion}

Human VASH2 gene is located on chromosome 1q32.3 and VASH2 protein is composed of 355 amino acid residues (6). It is reported that VASH2 can not only promote tumor growth and metastasis through supporting angiogenesis, but also regulate malignancies by direct effects on tumor cells. For the multiple functions in tumor progression, it has attracted more and more attention in recent years. In 2012, Takahashi et al proved that VASH2 could accelerate ovarian adenocarcinoma growth by promoting angiogenesis (14). In 2015, Kim et al reported that VASH2 was positively correlated with clinical stage, tumor proliferation and micro vessel density (MVD), as well as poor outcome of pancreatic cancer patients (9). For BC cancer, Tu et al reported that VASH 2 could promote proliferation and EMT of BC cells $(12,13)$. But, the role of VASH2 in drug resistance of $\mathrm{BC}$ cells has not been reported.

ADM is a classical chemotherapy drug and has been extensively used in BC patients. On one hand, ADM can inhibit DNA transcription and replication by intercalating between DNA base pairs; on the other hand, ADM can induce breakage of DNA double strands by generating oxygen free radicals (15). So, in the present study, we chose ADM to study drug resistance of BC cells. MDA-MB-231 and MCF-7 are both $\mathrm{BC}$ cell lines, but the original characteristics are not same. Previous studies proved that MDA-MB-231 cells were ER (-) and grew rapidly. Otherwise, MCF-7 cells were ER (+) and grew relatively slowly (16). Both of MDA-MB-231 and MCF-7 cell lines were used in our research. In this study, we found that BC cells with higher expression of VASH2 exhibited stronger ADM resistance. Then, overexpressing VASH2 increased ADM resistance, but silencing VASH2 inhibited ADM resistance in BC cells. Moreover, newly established ADM resistance cell line also showed stronger expression of VASH2 than parent cells. This confirmed promotion roles of $\mathrm{VASH} 2$ in ADM resistance of $\mathrm{BC}$ cells.

ABCG2 is also known as BC Resistance Protein (BCRP) and can function as one of the major factors inducing drug resistance of cancer cells. It can transport different chemotherapy drugs from intracellular region to extracellular space, thus providing drug resistance. So, ABCG2 has been treated as an important target for improving sensitivity of tumor cells to chemotherapy $(17,18)$. In the present study, we found that changes of VASH2 could induce consistent changes of ABCG2 after transfection. Moreover, silencing ABCG2 abrogated increase of ADM resistance induced by VASH2 partly, this proved that VASH2 could regulate ADM resistance of $\mathrm{BC}$ cells through regulating ABCG2, at least partly.

Deep understanding about molecular mechanism will contribute to find new therapy targets. AKT and ERK signal pathways can be stimulated in many types of tumors and can play important roles in tumor proliferation, migration, drug resistance, and radio resistance (19-21). In 2016, Hu CF reported that acidic microenvironment could induce drug resistance through upregulating expression of ABCG2 in lung cancer cells via PI3K-AKT-mTOR-S6 pathway (22). He et al reported that HIF-1 $\alpha$ could regulate ABCG 2 activity through the activation of ERK1/2 pathway and contribute to chemoresistance in pancreatic cancer cells (23). In the present study, we confirmed that VASH2 could promote expression of ABCG2 in BC cells via AKT signal pathway. This is in consistence with report from $\mathrm{Hu}$ et al (23), but different to report from He et al. This may be attributed to different function factors or different tumor types.

In conclusion, we confirmed the roles of VASH2 in ADM resistance of $\mathrm{BC}$ for the first time. Moreover, we proved that AKT-ABCG2 pathway was responsible for drug resistance induced by VASH2, at least partly. This contributes to our further understanding about VASH2 in tumor progression and suggests that VASH2 may be a novel target in $\mathrm{BC}$ treatment.

\section{References}

1. Clarke CA, Glaser SL, Leung R, Davidson-Allen K, Gomez SL and Keegan TH: Prevalence and characteristics of cancer patients receiving care from single vs. multiple institutions. Cancer epidemiol 46: 27-33, 2016.

2. Narayanan R and Dalton JT: Androgen receptor: A complex therapeutic target for breast cancer. Cancers (Basel) 8: pii: E108, 2016.

3. Friese CR, Li Y, Bondarenko I, Hofer TP, Ward KC, Hamilton AS, Deapen D, Kurian AW and Katz SJ: Chemotherapy decisions and patient experience with the recurrence score assay for early-stage breast cancer. Cancer 123: 43-51, 2017.

4. Cata JP, Chavez-MacGregor M, Valero V, Black W, Black DM, Goravanchi F, Ifeanyi IC, Hernandez M, Rodriguez-Restrepo A and Gottumukkala V: The impact of paravertebral block analgesia on breast cancer survival after surgery. Reg Anesth Pain Med 41: 696-703, 2016.

5. Mikkola TS, Savolainen-Peltonen H, Tuomikoski P, Hoti F, Vattulainen P, Gissler M and Ylikorkala O: Reduced risk of breast cancer mortality in women using postmenopausal hormone therapy: A Finnish nationwide comparative study. Menopause 23: 1199-1203, 2016.

6. Sato Y: The vasohibin family. Pharmaceuticals (Basel) 3: 433-440, 2010. 
7. Li Z, Tu M, Han B, Gu Y, Xue X, Sun J, Ge Q, Miao Y, Qian Z and Gao W: Vasohibin 2 decreases the cisplatin sensitivity of hepatocarcinoma cell line by downregulating p53. PLoS One 9: e90358, 2014.

8. Xue X, Zhang Y, Zhi Q, Tu M, Xu Y, Sun J, Wei J, Lu Z, Miao Y and Gao W: MiR200-upregulated Vasohibin 2 promotes the malignant transformation of tumors by inducing epithelial-mesenchymal transition in hepatocellular carcinoma. Cell Commun Signal 12: 62, 2014.

9. Kim JC, Kim KT, Park JT, Kim HJ, Sato Y and Kim HS: Expression of vasohibin-2 in pancreatic ductal adenocarcinoma promotes tumor progression and is associated with a poor clinical outcome. Hepatogastroenterology 62: 251-256, 2015.

10. Koyanagi T, Suzuki Y, Saga Y, Machida S, Takei Y, Fujiwara H, Suzuki M and Sato Y: In vivo delivery of siRNA targeting vasohibin-2 decreases tumor angiogenesis and suppresses tumor growth in ovarian cancer. Cancer Sci 104: 1705-1710, 2013.

11. Koyanagi T, Saga Y, Takahashi Y, Suzuki Y, Suzuki M and Sato Y: Downregulation of vasohibin-2, a novel angiogenesis regulator, suppresses tumor growth by inhibiting angiogenesis in endometrial cancer cells. Oncol Lett 5: 1058-1062, 2013.

12. Tu M, Liu X, Han B, Ge Q, Li Z, Lu Z, Wei J, Song G, Cai B, $\mathrm{Lv} \mathrm{N}$, et al: Vasohibin-2 promotes proliferation in human breast cancer cells via upregulation of fibroblast growth factor- 2 and growth/differentiation factor-15 expression. Mol Med Rep 10: 663-669, 2014.

13. Tu M, Lu C, Lv N, Wei J, Lu Z, Xi C, Chen J, Guo F, Jiang K, Li Q, et al: Vasohibin 2 promotes human luminal breast cancer angiogenesis in a non-paracrine manner via transcriptional activation of fibroblast growth factor 2. Cancer Lett 383: 272-281, 2016.

14. Takahashi Y, Koyanagi T, Suzuki Y, Saga Y, Kanomata N, Moriya T, Suzuki M and Sato Y: Vasohibin-2 expressed in human serous ovarian adenocarcinoma accelerates tumor growth by promoting angiogenesis. Mol Cancer Res 10: 1135-1146, 2012.
15. Zhao M, Yu S and Zhang M: Differential expression of multidrug resistance-related proteins in adriamycin-resistant (pumc-91/ADM) and parental (pumc-91) human bladder cancer cell lines. Mol Med Rep 14: 4741-4746, 2016.

16. Cetin I and Topcul MR: In vitro antiproliferative effects of nab-paclitaxel with liposomal cisplatin on MDA-MB-231 and MCF-7 breast cancer cell lines. J BUON 22: 347-354, 2017.

17. Sui H, Zhou LH, Zhang YL, Huang JP, Liu X, Ji Q, Fu XL, Wen HT, Chen ZS, Deng WL, et al: Evodiamine suppresses ABCG2 mediated drug resistance by inhibiting p50/p65 NF- $\mathrm{BB}$ pathway in colorectal cancer. J Cell Biochem 117: 1471-1481, 2016.

18. Sun Y, Gu M, Zhu L, Liu J, Xiong Y, Wei Y and Li F: Gemcitabine upregulates ABCG2/BCRP and modulates the intracellular pharmacokinetic profiles of bioluminescence in pancreatic cancer cells. Anticancer Drugs 27: 183-191, 2016.

19. Dahlmann M, Okhrimenko A, Marcinkowski P, Osterland M, Herrmann P, Smith J, Heizmann CW, Schlag PM and Stein U: RAGE mediates S100A4-induced cell motility via MAPK/ERK and hypoxia signaling and is a prognostic biomarker for human colorectal cancer metastasis. Oncotarget 5: 3220-3233, 2014.

20. Li N, Cui J, Duan X, Chen H and Fan F: Suppression of type I collagen expression by miR-29b via PI3K, Akt, and Sp1 pathway in human Tenon's fibroblasts. Invest Ophthalmol Vis Sci 53: $1670-1678,2012$.

21. Miao B and Degterev A: Targeting phospshatidylinositol 3-kinase signaling with novel phosphatidylinositol 3,4,5-triphosphate antagonists. Autophagy 7: 650-651, 2011.

22. Hu CF, Huang YY, Wang YJ and Gao FG: Upregulation of ABCG2 via the PI3K-Akt pathway contributes to acidic microenvironment-induced cisplatin resistance in A549 and LTEP-a-2 lung cancer cells. Oncol Rep 36: 455-461, 2016.

23. He X, Wang J, Wei W, Shi M, Xin B, Zhang T and Shen X: Hypoxia regulates ABCG2 activity through the activivation of ERK1/2/HIF-1 $\alpha$ and contributes to chemoresistance in pancreatic cancer cells. Cancer Biol Ther 17: 188-198, 2016. 